Systematic gas gain measurements and Penning energy transfer rates in $\mathrm{Ne}-\mathrm{CO}_{2}$ mixtures

This content has been downloaded from IOPscience. Please scroll down to see the full text. 2016 JINST 11 P01003

(http://iopscience.iop.org/1748-0221/11/01/P01003)

View the table of contents for this issue, or go to the journal homepage for more

Download details:

IP Address: 131.169.4.70

This content was downloaded on 07/03/2016 at 22:19

Please note that terms and conditions apply. 


\section{Systematic gas gain measurements and Penning energy transfer rates in $\mathrm{Ne}-\mathrm{CO}_{2}$ mixtures}

\section{Ö. Şahin, ${ }^{a, 1}$ T.Z. Kowalski ${ }^{b}$ and R. Veenhof ${ }^{a, c}$}

${ }^{a}$ Department of Physics, Uludă̆ University, 16059 Bursa, Turkey

${ }^{b}$ Faculty of Physics and Applied Computer Science, AGH University of Science and Technology, Kraków, Poland

${ }^{c}$ RD51 collaboration, CERN,

Genève, Switzerland

E-mail: osahin@uludag.edu.tr

Abstract: In Ne- $\mathrm{CO}_{2}$ mixtures, excitation energy of $\mathrm{Ne}$ atom can be used to ionize $\mathrm{CO}_{2}$ molecule by the mechanisms called Penning transfers. In the present work, we have measured the gas gain systematically in various $\mathrm{Ne}-\mathrm{CO}_{2}$ mixtures $\left(\mathrm{Ne}+0.6-60 \% \mathrm{CO}_{2}\right)$ at $0.4,0.8,1.2,1.8 \mathrm{~atm}$. The experimental data have been fitted to investigate the Penning energy transfer rates and the secondary processes playing a role in avalanche formations.

Keywords: Avalanche-induced secondary effects; Gaseous detectors; Charge transport and multiplication in gas; Detector modelling and simulations I (interaction of radiation with matter, interaction of photons with matter, interaction of hadrons with matter, etc)

\footnotetext{
${ }^{1}$ Corresponding author.
} 


\section{Contents}

1 Introduction $\quad 1$

1.1 Excitation induced ionizations 2

2 Gas gain measurements $\quad 4$

3 Analysis of gas gain data $\quad 5$

3.1 Obtaining Penning energy transfer rates 5

3.2 Effective correction parameter for the secondary processes 5

4 Outcome of data analysis $\quad 6$

4.1 Simulation of the measured gas gain data 6

$\begin{array}{lll}4.2 & \text { Penning energy transfer rates } & 8\end{array}$

$\begin{array}{lll}4.3 & \text { Effective correction parameters } & 8\end{array}$

5 Conclusions $r$

$\begin{array}{lr}\text { A Measurements and simulations } & 10\end{array}$

\section{Introduction}

$\mathrm{Ne}-\mathrm{CO}_{2}$ was chosen as an operating gas mixture for the ALICE TPC since it leads high ionization rate, high ion mobility, low electron diffusion, saturated drift velocity, slow ageing etc. [1-4] The gas gain measurements for the ALICE TPC were made in Ne with $7.9-13.8 \% \mathrm{CO}_{2}$ mixtures at 990-1010 mbar pressures [5]. The uncertainty of the $\mathrm{CO}_{2}$ concentration for the measurements was reported as $\pm 0.5 \%$ (absolute) [6]. In addition, the range of the gas gain was limited to $210^{3}-510^{4}$. The parallel ionization multiplier (PIM) and micromegas detector with different amplification gap distances were used to measure the gas gain in $\mathrm{Ne} 90 \% \mathrm{CO}_{2} 10 \%$ mixture [7, 8]. The limited range of the gas gain, the lack of data for low and high $\mathrm{CO}_{2}$ fractions at different mixture pressures and the experimental deficiencies prevent to get accurate information from these measurements about the additional processes involved in electron avalanche growth.

In the present work, we have measured the gas gain curves in a wide range from the ionization chamber regime to the breakdown limit ${ }^{1}$ in $\mathrm{Ne}-\mathrm{CO}_{2}$ gas compositions $\left(0.6-60 \% \mathrm{CO}_{2}\right.$, also in pure $\left.\mathrm{CO}_{2}\right)$ at various mixture pressures $(0.4,0.8,1.2,1.8 \mathrm{~atm})$. The measured gas gain data have been fitted with a developed tool using output files of Magboltz [9] simulation program to calculate the Penning transfer rates. The secondary processes related to electron avalanche development have been also investigated.

${ }^{1}$ Breakdown limit — a deviation relative to an exponential increase. 


\subsection{Excitation induced ionizations}

Collision of a free electron with a neutral noble gas atom $(A)$ may cause the following basic mechanisms:

$$
\begin{aligned}
& e^{-}+A \rightarrow e^{-}+e^{-}+A^{+} \\
& e^{-}+A \rightarrow e^{-}+A^{*} .
\end{aligned}
$$

In an avalanche development, besides the Townsend (direct) ionization (1.1), excitation levels $\left(A^{*}\right)$ can be formed (1.2). In the mixtures containing an admixture with ionization potential lower than the excited states of the noble gas, additional ionization processes can lead to electron enhancements. Contribution of the excited states to the direct ionization is known as Penning transfers:

$$
A^{*}+B \rightarrow A+B^{+}+e^{-}
$$

where $B$ and $B^{+}$are the ground state and the ionized atom (molecule) of the admixture gas, respectively.

Numerous mechanisms have been proposed for the excited state energy transfers; ionization resulting from the radiative decay of the excited state, electron exchange [10] or direct electron transfer during collision [11, 12], associative ionization [13] and excimer formation [14, 15]. The effect of such excitation induced ionizations on the gas gain is called "Penning effect" after Frans Michel Penning who observed that the discharge potential in pure neon, argon and mercury are higher than in their mixtures [16-18]. It is reported that the resolution of gas detectors can be improved considerably by using Penning mixtures since the Penning effect reduces both the mean energy required to produce ion pairs and the Fano factor [19-21].

In a cylindrical counter, the electron avalanches develop generally at a few wire radii called multiplication region in which the electrons can attain sufficient energy from the electric field between the collisions to initiate new ionizations. Production point of the additional electrons is an important criteria to define the effect of the energy transfers on gas gain enhancements [22, 23]:

- Region 1: Additional electrons via the mechanism (1.3) can be created in the same place where the excited noble gas atoms are created, at a distance smaller than $1 / \alpha$ ( $\alpha$ is the Townsend coefficient). In this case, the effect of these extra electrons on gas gain can not be distinguished from those produced in the Townsend ionization process (1.1).

- Region 2: The extra electron formations can take place at a distance from the point where excitations occurred but again in the multiplication region. The effect of such additional electrons on avalanche multiplication is smaller than those produced in the impact ionization zone since the electric field in the cylindrical chamber becomes non-uniformly weaker away from the anode wire.

- Region 3: If the extra electrons are generated outside the multiplication volume (drift region) or on the cathode surface, then they are fully multiplied while moving to the anode. The contribution of such avalanche electrons leads to over-exponential increases on the gas gain curves. 
The reduced electric field strength $S$, in a cylindrical counter, is given by:

$$
S(r)=\frac{E(r)}{p}=\frac{V_{\mathrm{a}}}{p r \ln \left(\frac{r_{\mathrm{c}}}{r_{\mathrm{a}}}\right)},
$$

where $E(r)$ is the electric field strength at a radial distance $r$ from the center of the anode wire, $p$ is the gas pressure, $V_{\mathrm{a}}$ is the anode potential, $r_{\mathrm{c}}$ and $r_{\mathrm{a}}$ are the radius of the cathode and the anode, respectively. The avalanche size of the electrons can be determined using the formula of Diethorn [24] and its practical form proposed by Zastawny [25]:

$$
\frac{\ln G}{p r_{\mathrm{a}} S_{\mathrm{a}}}=\frac{\ln 2}{W} \ln S_{\mathrm{a}}-\frac{\ln 2}{W} \ln H,
$$

here $G$ refers to the gas gain, $S_{\mathrm{a}}$ is the reduced electric field at the anode surface, $W$ corresponds to the average kinetic energy of the electrons gained between two successive ionizing collisions and $H$ is the value of the reduced electrical field strength at which the multiplication starts. Characteristic gas mixture constants, $W$ and $H$, can be extracted from the experimental gas gains. If the coordinates are chosen as $\ln G /\left(p r_{\mathrm{a}} S_{\mathrm{a}}\right)$ vs. $\ln S_{\mathrm{a}}$, then the gas gain data should lie on a straight line. Therefore, the constants $W$ and $H$ can be determined from the slope and intercept of a linear fit function. According to eq. (1.5) for $S_{\mathrm{a}}=H$ the gas gain becomes $G=1$. Substituting $H$ in eq. (1.4) the radius of the avalanche $\left(r_{\mathrm{G}=1}\right)$ for given anode potential (corresponding to the measured gas gains) can be defined as:

$$
r_{\mathrm{G}=1}=\frac{V_{\mathrm{a}}}{H p \ln \left(\frac{r_{\mathrm{c}}}{r_{\mathrm{a}}}\right)} .
$$

Where, $r_{\mathrm{G}=1}$ is the radius in which the avalanche multiplication of electrons develops from the gas gain of $G=1$ to the current values for the applied voltages. The potential $V_{\mathrm{G}=2}$, for which the gas gain is $G=2$, can be calculated with the interpolation of the gas gain measurements. Then, using eq. (1.4) the reduced electric field is found:

$$
S_{\mathrm{G}=2}=\frac{V_{\mathrm{G}=2}}{p r_{\mathrm{a}} \ln \left(\frac{r_{\mathrm{c}}}{r_{\mathrm{a}}}\right)} .
$$

By analogy with eq. (1.6), and replacing $H$ with $S_{\mathrm{G}=2}$, the radius of the avalanches occurring from $G=2$ to the current values for the applied anode potentials is:

$$
r_{\mathrm{G}=2}=\frac{V_{\mathrm{a}}}{S_{\mathrm{G}=2} p \ln \left(\frac{r_{\mathrm{c}}}{r_{\mathrm{a}}}\right)} .
$$

Example calculations of $r_{\mathrm{G}=1}$ and $r_{\mathrm{G}=2}$ as the function of gas gain at 0.4 and $1.8 \mathrm{~atm}$ are shown on figure 1. The pink bands refer to the ionization region, an intermediate zone between the electron multiplication and the drift region. In this zone, the production of the electron-impact excitation and ionization processes compete with each other since the electric field strength is radially smaller than the multiplication region (see, eq. (1.4)). The depth of the zone, $r_{\mathrm{G}=1}-r_{\mathrm{G}=2}$ (red lines on figure 1), is getting significantly larger with the decrease of the mixture pressure. This indicates that the diameter of the "Region 2", described above, will be bigger at lower pressures. As a result, the probability of the excitation induced ionizations for the same $\mathrm{CO}_{2}$ concentration is likely to rise with the increasing mixture pressure. 

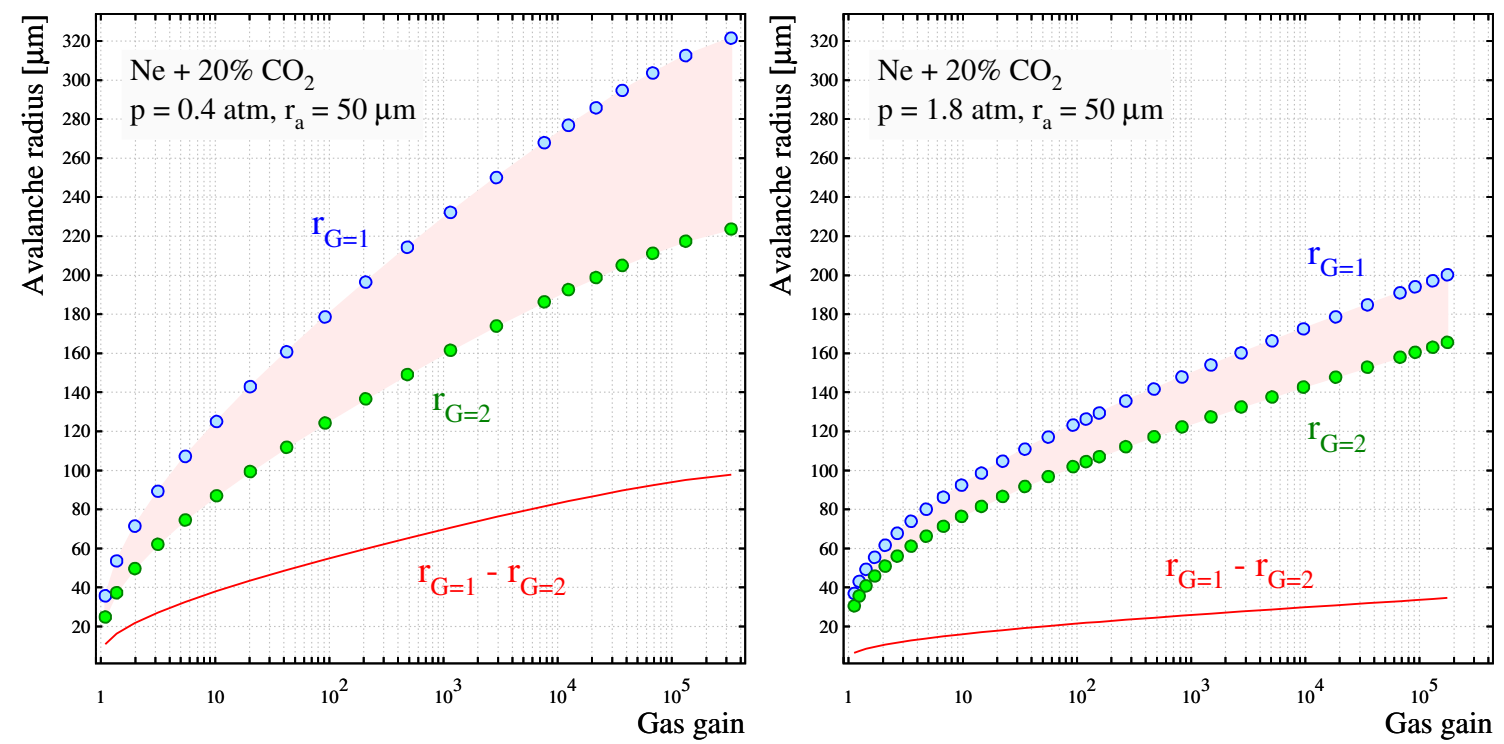

Figure 1. Radius of the avalanche in neon with $20 \% \mathrm{CO}_{2}$ gas mixture at 0.4 atm (left) and 1.8 atm (right) pressures. The blue and green circles $\left(r_{\mathrm{G}=1}\right.$ and $\left.r_{\mathrm{G}=2}\right)$ - the radius from $G=1$ and $G=2$ to the current values corresponding to the applied anode voltages; calculated using eq. (1.6) and eq. (1.8), respectively. The pink bands - the zone between $r_{\mathrm{G}=1}$ and $r_{\mathrm{G}=2}$; the red lines - the depth of the zone in which the gas gain increases from $G=1$ to $G=2$.
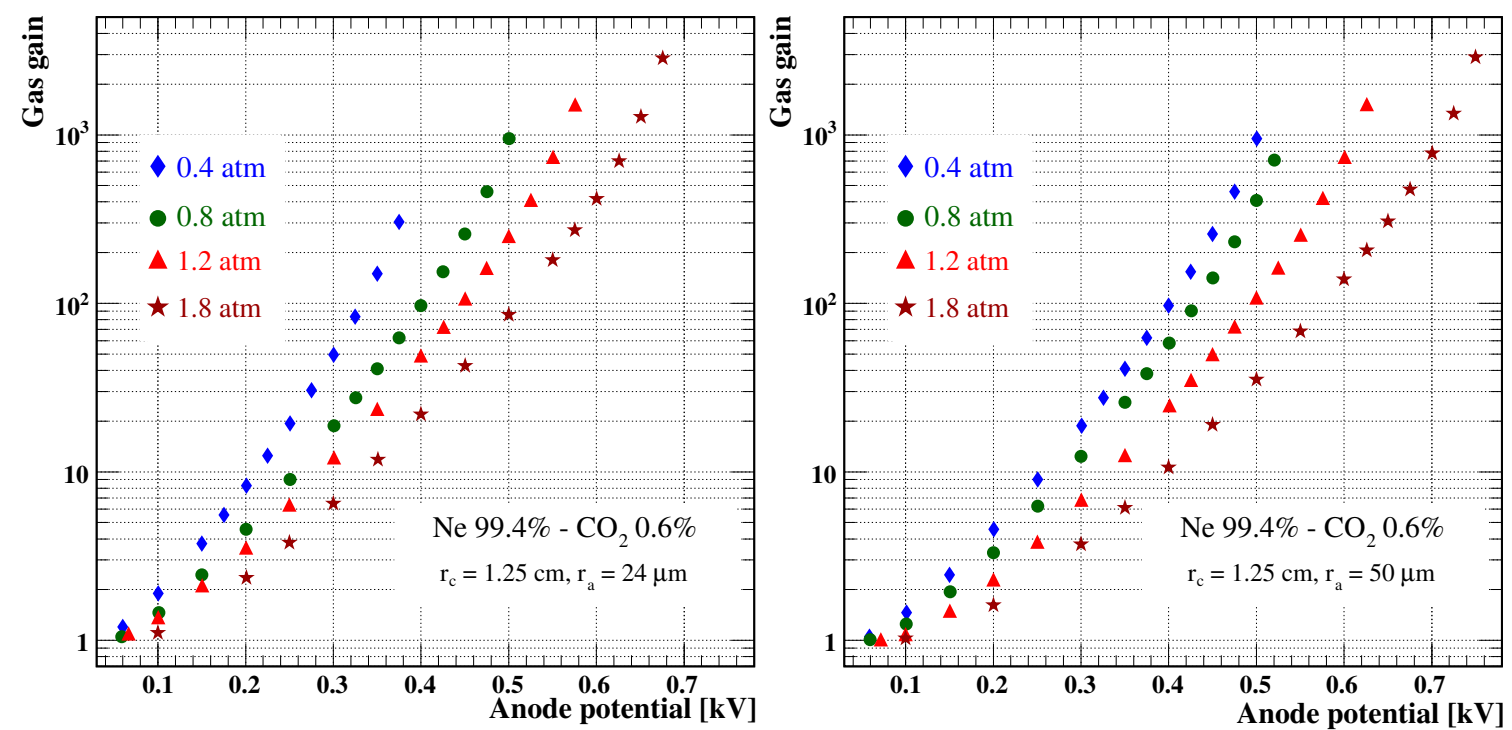

Figure 2. Examples of the measured gas gain curves in different cylindrical chambers for the lowest $\mathrm{CO}_{2}$ percentage considered in this work.

\section{Gas gain measurements}

The gas gains were measured in cylindrical chambers filled with thirteen different $\mathrm{Ne}-\mathrm{CO}_{2}$ mixtures. The cathode radius of the chambers was the same $\left(r_{\mathrm{c}}=1.25 \mathrm{~cm}\right)$ and anode wires with a radius $r_{\mathrm{a}}$ of $24 \mu \mathrm{m}$ or $50 \mu \mathrm{m}$ were placed in the center (figure 2). 
Neon of purity 5.0 and $\mathrm{CO}_{2}$ of purity 4.8 were used in the measurements. The gas mixtures were prepared with less than $1 \%$ error on the mixing proportions. The gas gain was determined as the ratio of the current value at given voltage to the current in the ionization chamber regime (the current method) [26]. Therefore the error of the measured gas gain was dominated by the uncertainties on the currents. Consequently, absolute accuracy of the measured gain data was better than $5 \%$.

\section{Analysis of gas gain data}

The measured gas gain curves were simulated with two fit parameters. The main parameter was the Penning energy transfer rate and the second fit parameter was used to define over-exponential increase in the gas gain curves.

\subsection{Obtaining Penning energy transfer rates}

Penning transfer adjusted gas gain $G$ for a single wire tube is given by:

$$
G=\exp \int_{r_{\mathrm{m}}}^{r_{\mathrm{a}}} \alpha_{\text {Pen }}(E(r)) \mathrm{d} r
$$

here $r$ is the radial distance of the avalanche electrons from the anode wire, $r_{\mathrm{m}}$ is the starting point of the electron multiplications at where $\alpha>0$ and $E(r)$ is the electric field. Penning energy transfer correction for the Townsend coefficient " $\alpha_{\text {Pen }}$ " is defined with the following expression [26, 27]:

$$
\alpha_{\text {Pen }}:=\alpha\left(1+r_{\text {Pen }} \frac{f_{\mathrm{Ne}}^{\mathrm{exc}}}{f_{\mathrm{mix}}^{\text {ion }}}\right),
$$

where

$f_{\text {mix }}^{\text {ion }}$ and $f_{\mathrm{Ne}}^{\text {exc }}$ : the total frequencies (production rates) of the direct ionizations $\left(\mathrm{Ne}^{+}\right.$and $\left.\mathrm{CO}_{2}^{+}\right)$, proportional to $\alpha$, and the excited neon states, respectively.

$r_{\text {Pen }}$ : Penning transfer rate $r_{\text {Pen }}$, the probability that an excited neon atom ionizes a $\mathrm{CO}_{2}$ molecule.

The lowest excited state of neon $\left(2 p^{5} 3 s, 16.619 \mathrm{eV}\right)$ has larger energy than the ionization potential of $\mathrm{CO}_{2}(13.773 \mathrm{eV})$. Therefore, all the excited states of neon located below the ionization threshold are eligible for transferring energy to ionize $\mathrm{CO}_{2}$ molecules.

\subsection{Effective correction parameter for the secondary processes}

The excited neon atoms $\left(\mathrm{Ne}^{*}\right)$ and their molecular forms (excimers, $\mathrm{Ne}_{2}^{*}$ ) may decay by photon emissions when they can not spend their excess energy in the inelastic collisions. If the quencher gas $\left(\mathrm{CO}_{2}\right)$ fraction in the gas mixture is not high enough to absorb these photons sufficiently, then they can reach to the drift region which is far from the main multiplication zone or to the cathode. The work functions of the metals used as cathode are in the range of $4-5 \mathrm{eV}$ [28]. Therefore, the UV photons released by the direct decays to the ground can create photo-electrons both from the $\mathrm{CO}_{2}$ molecules and at the cathode surface. However, the low-energy photons $(4-5 \mathrm{eV})$, emitted by the decays to the intermediate energy levels, can eject electrons only from the cathode. 

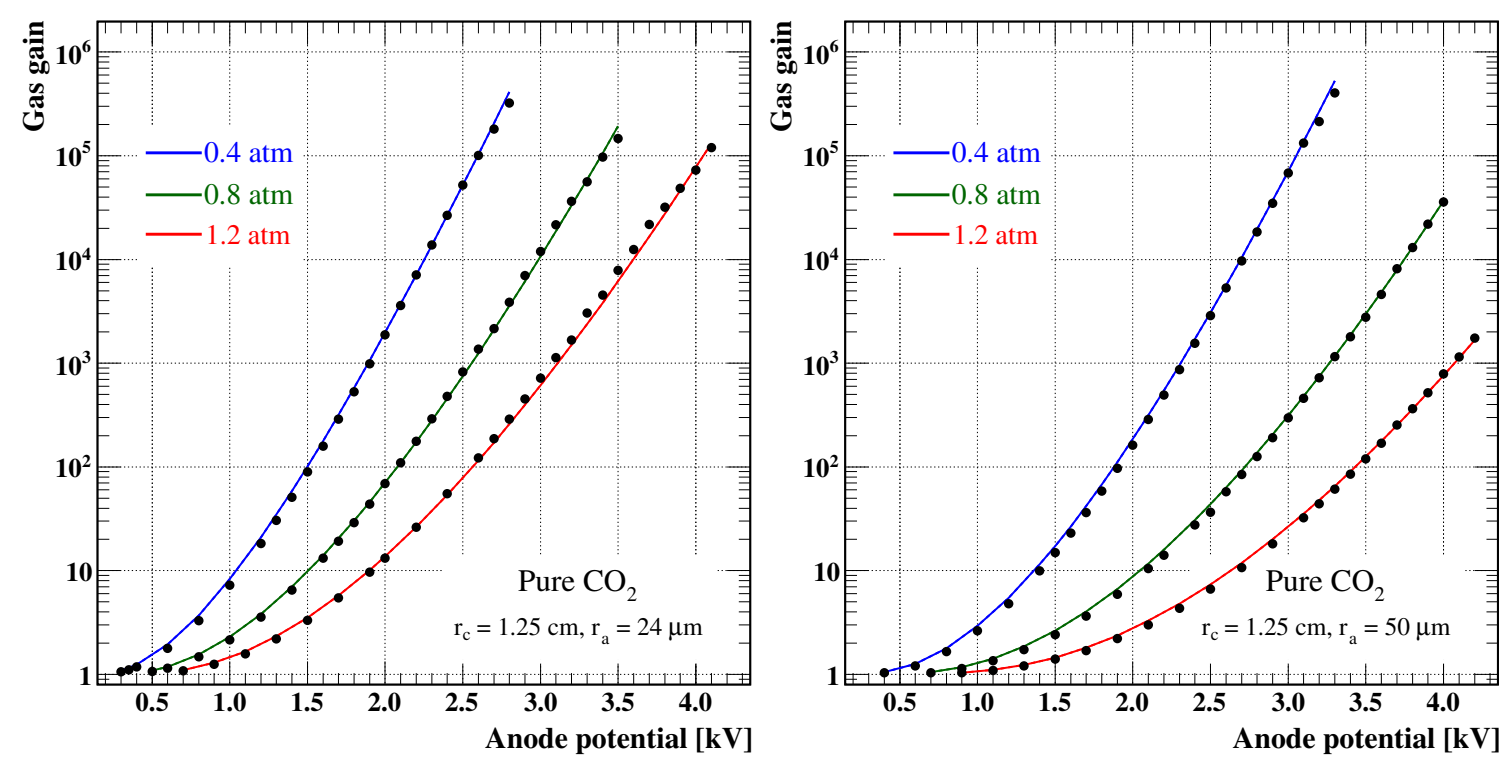

Figure 3. Calculated and measured gas gain curves in pure $\mathrm{CO}_{2}$ for different tubes. The lines are computed with Magboltz and the measured data are shown with the black points.

Neon ions created in the drift volume of the chamber may arrive at the cathode if they can gain sufficient energy from the electric field. So, the neutralization of the ion at the cathode surface can also cause an electron formation.

All those additional electrons will have their own avalanche cycles while moving to the anode wire. Such secondary avalanches result in an over-exponential increase in the gas gain curve. Production of the additional electron can be described with a single parameter $\beta[21,26,27,29]$ and the overall gas gain $G_{\mathrm{T}}$ is written as:

$$
G_{\mathrm{T}}=\frac{G}{1-\beta G}
$$

Since the effective parameter $\beta$ was not strongly correlated with the Penning transfer rate $r_{\text {Pen }}$, we could separate these parameters in the gas gain simulations.

\section{Outcome of data analysis}

\subsection{Simulation of the measured gas gain data}

The gas gain curves for pure $\mathrm{CO}_{2}$ calculated from the integration of the Townsend coefficients (computed by Magboltz 10.10) are in agreement with the experimental data (figure 3). This manifests that $\mathrm{CO}_{2}^{+}$cross sections used in Magboltz are correct.

The gas gain fits for $\mathrm{Ne} 99 \%-\mathrm{CO}_{2} 1 \%$ and $\mathrm{Ne} 98 \%-\mathrm{CO}_{2} 2 \%$ mixtures are shown on figure 4 . The dashed lines are calculated from the integration of the Townsend coefficients. The thin lines are the fits derived from the Penning adjusted Townsend coefficients $\alpha_{\text {Pen }}$; effective parameters for the secondaries are not taken into account in these fits. The thick lines represent the final fit results in which both the Penning adjustment and the effective parameter $(\beta)$ are included. 

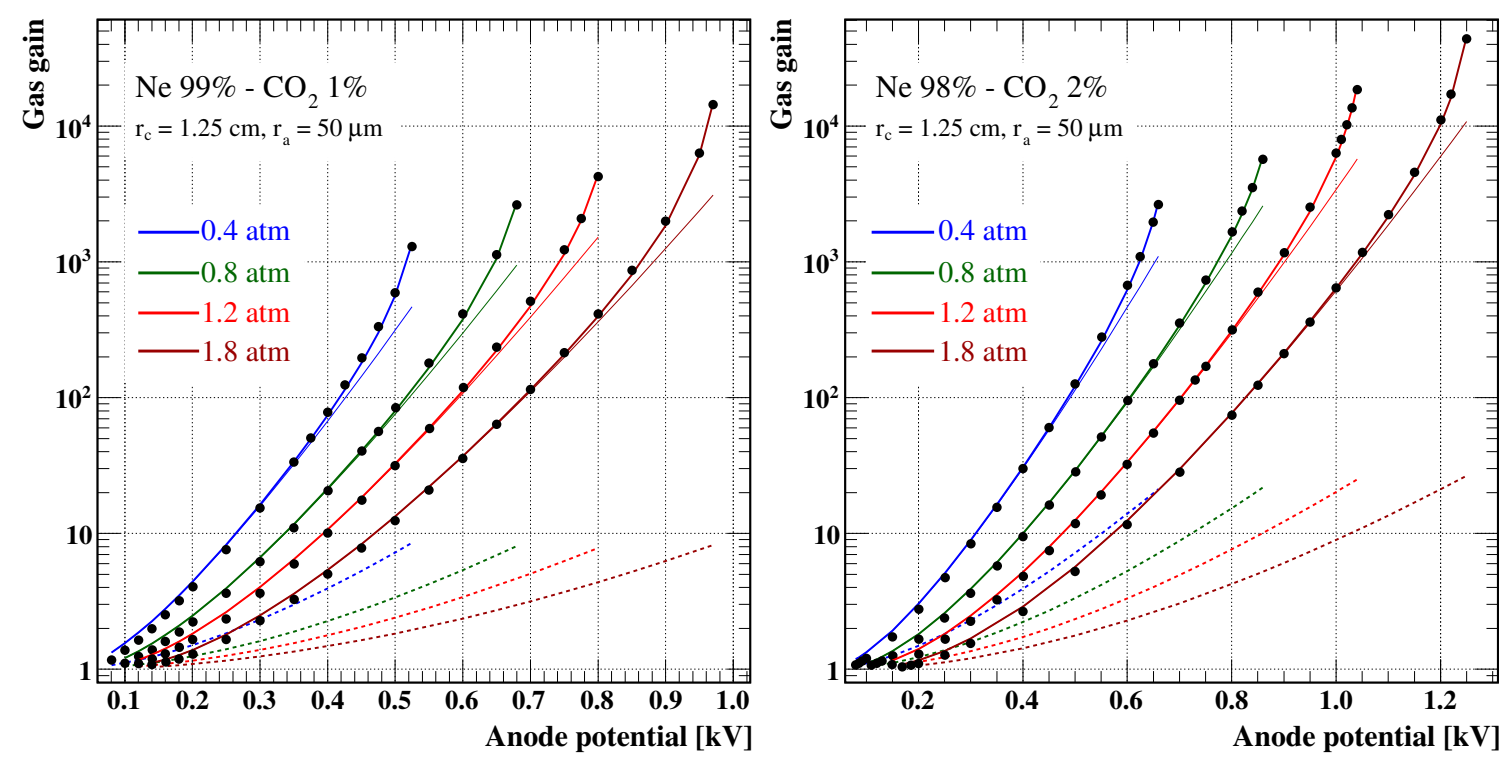

Figure 4. Calculated and measured gas gain curves. The dashed lines - calculated without any corrections; the thin lines - Penning transfer included; the thick lines - Penning transfer and effective parameter included; the black points - measured data.
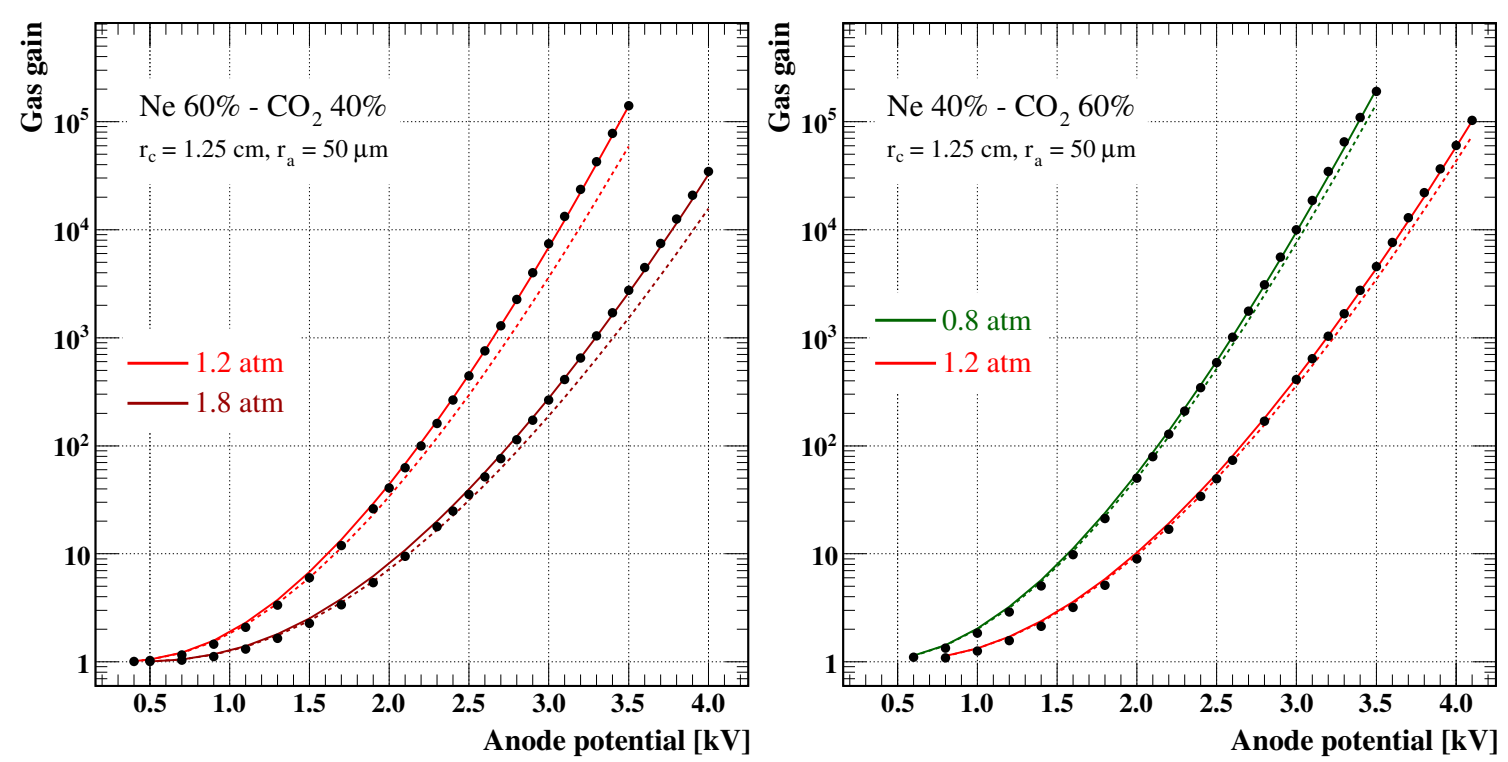

Figure 5. Calculated and measured gas gain curves. The dashed lines - calculated without any corrections; the thick lines - Penning transfer included; the black points - measured data.

The same calculation method is applied to the each experimental gas gain data to extract the Penning transfer rates and the effective parameters (see, appendix A). However, the second fit parameter $(\beta)$ is only used when there is over-exponential increase in the measured gas gain curves. As an example, the final fit results on figure 5 are obtained without including effective parameters. 


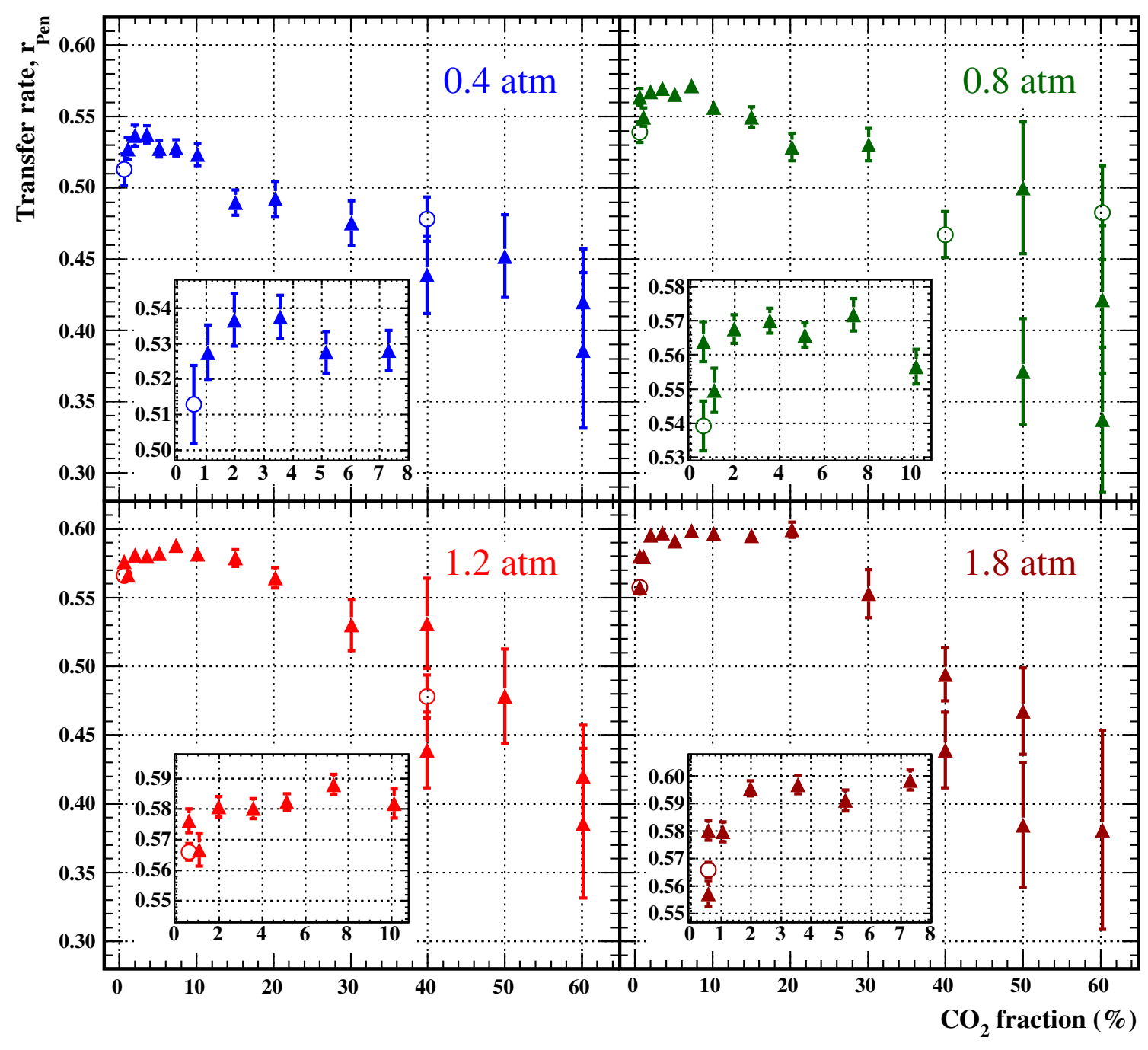

Figure 6. Penning energy transfer rates, $r_{\mathrm{Pen}}$, in $\mathrm{Ne}-\mathrm{CO}_{2}$ as the function of $\mathrm{CO}_{2}$ concentration at mixture pressures of 0.4, 0.8, 1.2, $1.8 \mathrm{~atm}$.

\subsection{Penning energy transfer rates}

The energy transfer rates $\left(r_{\text {Pen }}\right)$ calculated from the fits of the present gas gain measurements using eq. (3.2) are shown on figure 6. The triangles and open circles represent the transfer rates extracted from the gas gain data measured in tubes with anode radius of $50 \mu \mathrm{m}$ and $r_{\mathrm{a}}=24 \mu \mathrm{m}$, respectively.

\subsection{Effective correction parameters}

The effective parameters $(\beta)$ derived from the fits of the measured gas gain curves are shown on figure 7 (left). The decrease of $\beta$ with increasing pressure and $\mathrm{CO}_{2}$ fraction can be related to the photo-absorption cross sections of photons $\left(\sigma_{\mathrm{pa}}\right)$ emitted from excited neon atoms (figure 7 , right). At high mixture pressures and $\mathrm{CO}_{2}$ percentages the mean free path of photons will gradually be short; 

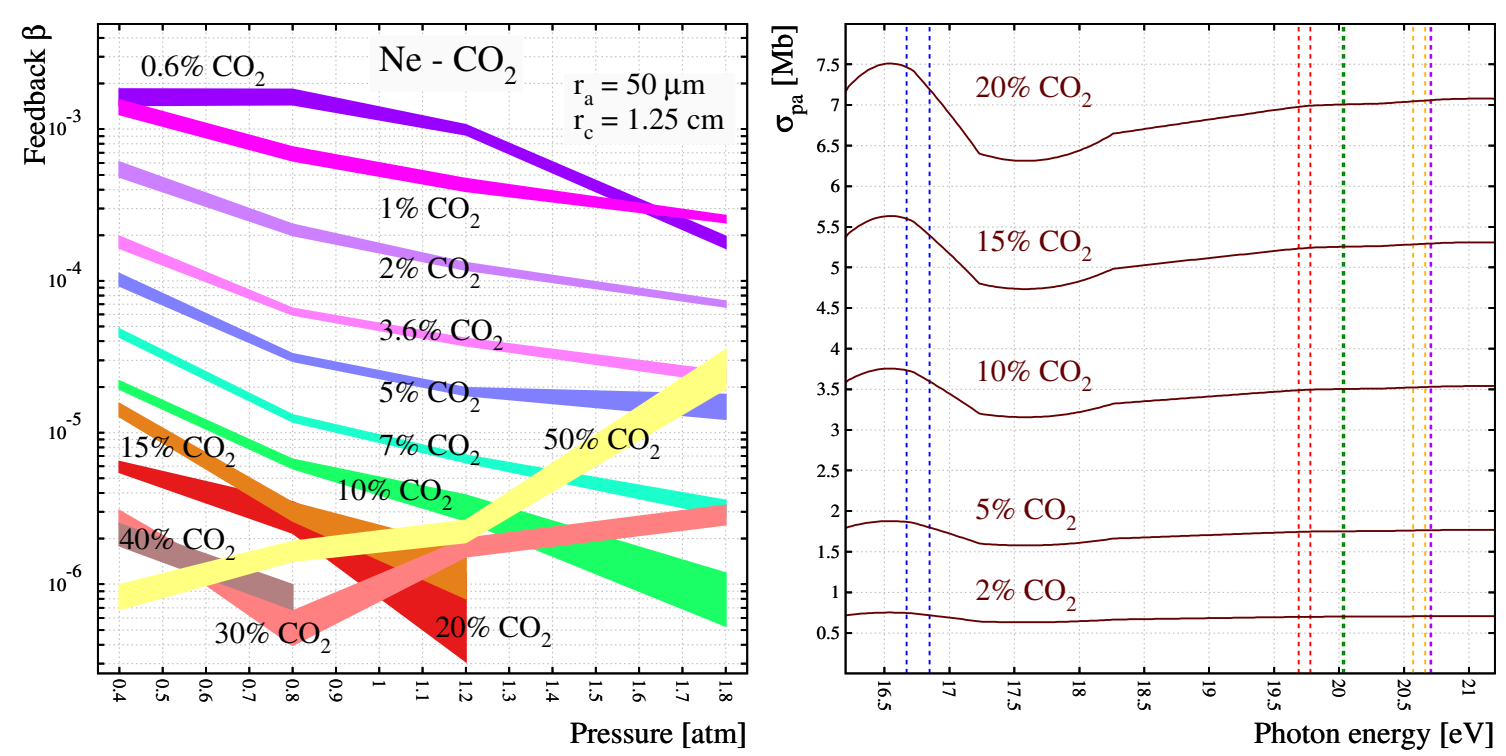

Figure 7. Left: effective parameters $\beta$ extracted from a fit to the experimental data. Calculated with eq. (3.3). The precision of the parameters are shown by bands. Right: photo-absorption cross section of $\mathrm{CO}_{2}\left(\sigma_{\mathrm{pa}}\right)$, compiled from J. Berkowitz [30] and the references therein. The dashed lines correspond to the energies of neon radiative states.

hence, the secondary avalanches leading the over-exponential growth of the gas multiplications are expected to decrease [29].

The gas gain distribution, the number of neon excited states for each gas mixture close to the breakdown, production and propagation mechanisms of the photons, neutralization of the positive ions at the cathode surface are other important arguments to identify $\beta$ parameters. In addition, the effective parameter surprisingly rises with the pressure in $30 \%$ (beyond $0.8 \mathrm{~atm}$ ) and $50 \% \mathrm{CO}_{2}$ mixtures. All these quantitative justifications are interest of a future work.

\section{Conclusions}

Gas composition and pressure dependence of the excitation induced ionization mechanisms in $\mathrm{Ne}-\mathrm{CO}_{2}$ mixtures have been investigated using the high-precision gas gain data measured with the cylindrical single wire chambers. The Penning energy transfer rates drop at high $\mathrm{CO}_{2}$ concentrations. The drops indicate that the excited states of neon are lost. The over-exponential increase of the gas gain due to the secondary avalanche processes has been described with an effective correction parameter.

\section{Acknowledgments}

This work was funded by the Turkish Atomic Energy Authority (2013 TAEK CERN-A5.H2.P1.0123), supported partially by the Scientific Research Projects Unit of Uludağ University with the project no BUAP(F)-2014/4 and by the Polish National Science Centre under the Grant DEC2013/10/M/ST7/00568. 


\section{A Measurements and simulations}
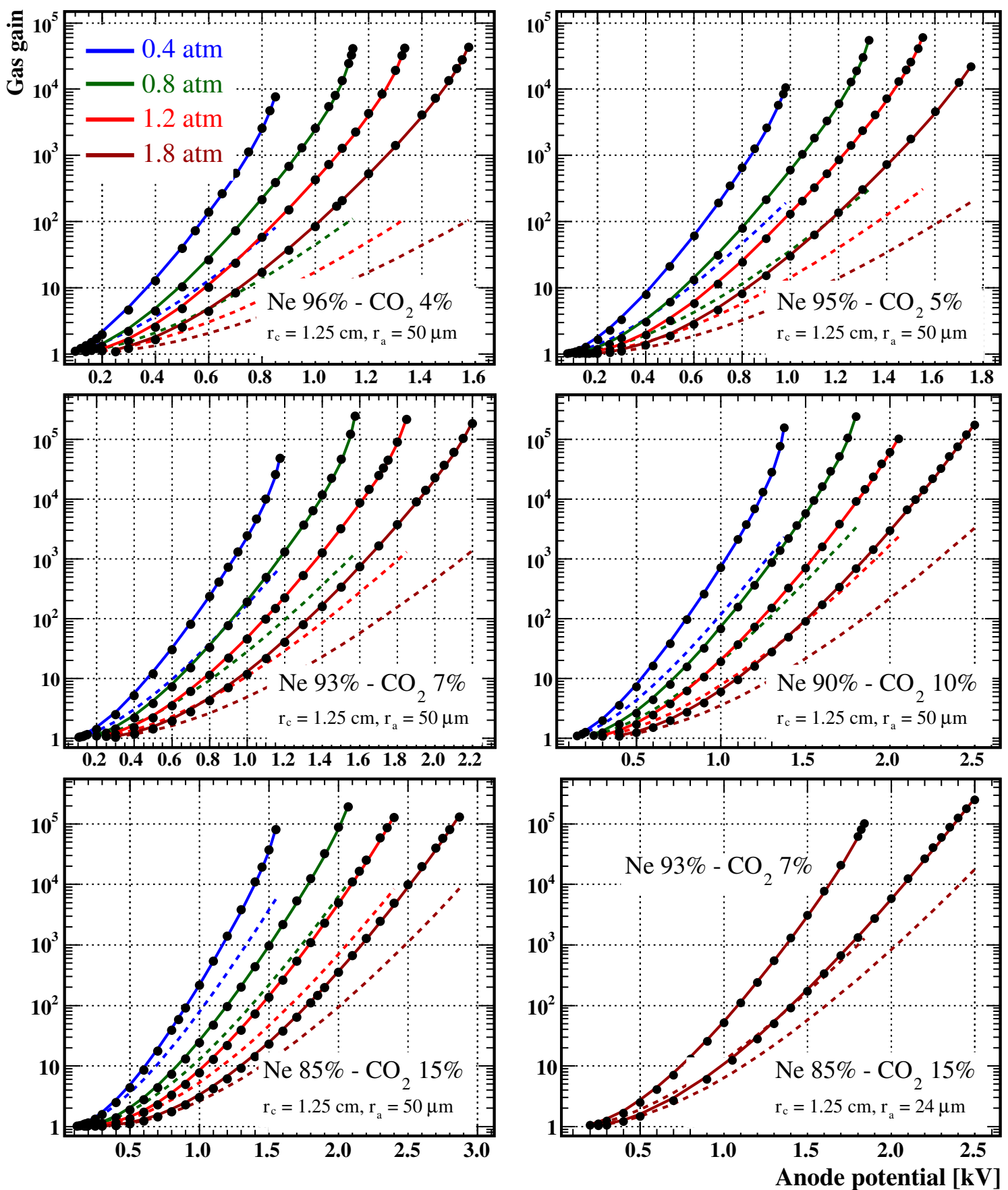

Figure 8. Measured and simulated gas gain curves in $\mathrm{Ne}+4 \%, 5 \%, 7 \%, 10 \%$ and $15 \% \mathrm{CO}_{2}$ mixtures at different pressures. The dashed lines are calculated with uncorrected Townsend coefficients. The lines are the fits with adjusted Townsend coefficients for Penning transfers; the over-exponential increases are fitted using effective parameter $\beta$. The black points show the experimental data. 

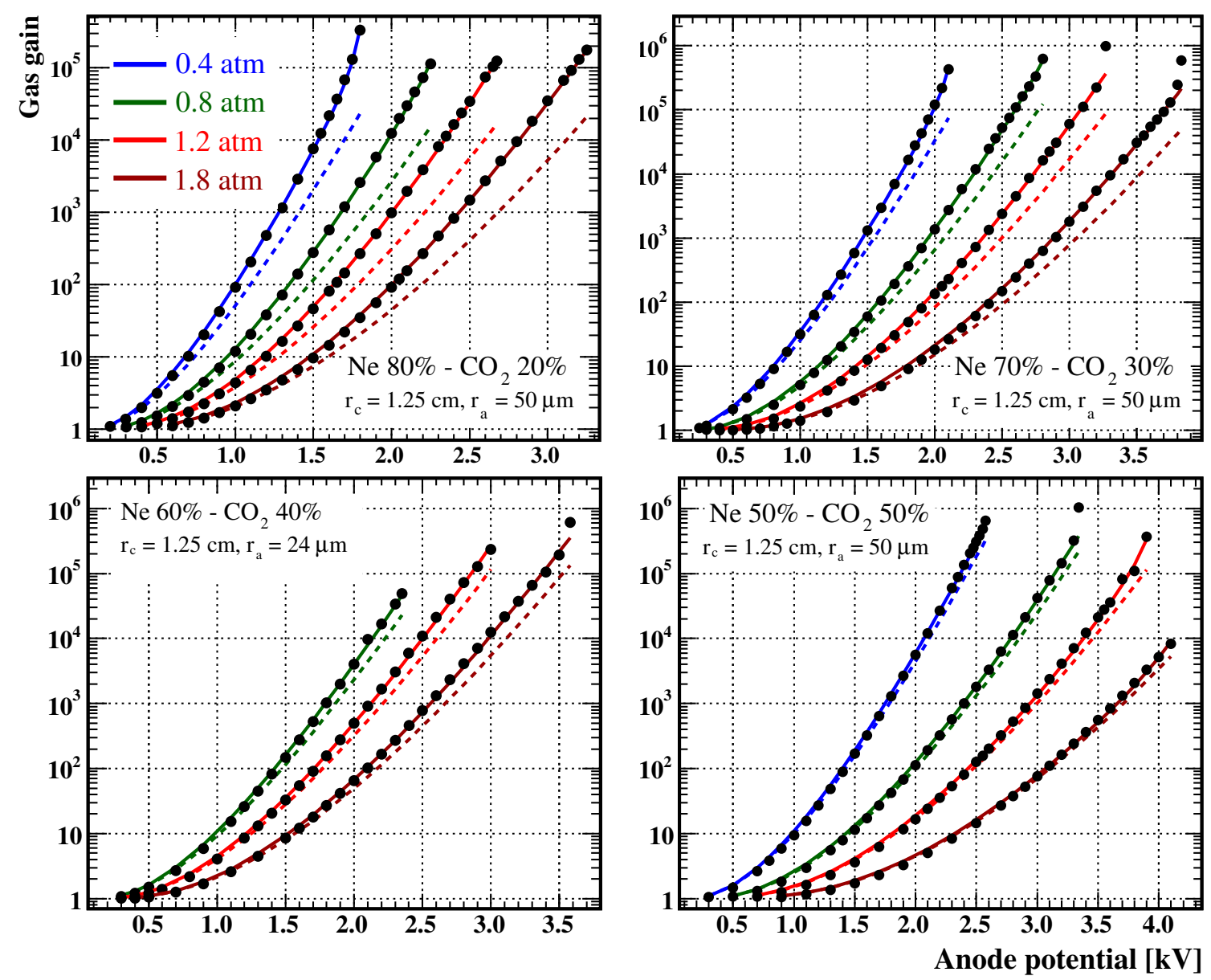

Figure 9. Measured and simulated gas gain curves in $\mathrm{Ne}+20 \%, 30 \%, 40 \%$ and $50 \% \mathrm{CO}_{2}$ mixtures at different pressures.

\section{References}

[1] ALICE collaboration, ALICE Time Projection Chamber: technical design report, CERN-LHCC-2000-001, CERN, Geneva Switzerland (2000).

[2] J. Alme et al., The ALICE TPC, a large 3-dimensional tracking device with fast readout for ultra-high multiplicity events, Nucl. Instrum. Meth. A 622 (2010) 316 [arXiv: 1001.1950].

[3] ALICE collaboration, Upgrade of the ALICE Time Projection Chamber, CERN-LHCC-2013-020, CERN, Geneva Switzerland (2013).

[4] R. Veenhof, Choosing a gas mixture for the ALICE TPC, ALICE-INT-2003-29, version 1.0, CERN, Geneva Switzerland (2003).

[5] ALICE collaboration, C. Garabatos, The ALICE TPC, Nucl. Instrum. Meth. A 535 (2004) 197.

[6] C. Garabatos, private communication, August 202010.

[7] D. Thers et al., New prospects on particle detection with a Parallel Ionization Multiplier (PIM), Nucl. Instrum. Meth. A 535 (2004) 562.

[8] J. Wiechula et al., Parallel Ionization Multiplier: a gaseous detector dedicated to the tracking of minimum ionization particles, Nucl. Instrum. Meth. A 573 (2007) 294. 
[9] S.F. Biagi, Monte Carlo simulation of electron drift and diffusion in counting gases under the influence of electric and magnetic fields, Nucl. Instrum. Meth. A 421 (1999) 234.

[10] H. Hotop and A. Niehaus, Reactions of excited atoms and molecules with atoms and molecules, II. Energy analysis of Penning electrons, Z. Phys. 228 (1969) 66.

[11] K. Katsuura, Ionization of atoms by collisions with excited atoms, J. Chem. Phys. 42 (1965) 3771.

[12] B.M. Smirnov and O.B. Firsov, Ionization of an atom colliding with an excited atom, JETP Pisma 2 (1965) 478 .

[13] J.A. Hornbeck and J.P. Molnar, Mass spectrometric studies of molecular ions in the noble gases, Phys. Rev. 84 (1951) 621.

[14] T. Oka et al., Energy transfer of argon excited diatomic molecules, J. Chem. Phys 70 (1979) 3384.

[15] W. Wieme and J. Lenaerts, Excimer formation in argon, krypton, and xenon discharge afterglows between 200 and 400 K, J. Chem. Phys. 74 (1981) 483.

[16] F.M. Penning, Über den Einfluß sehr geringer Beimischungen auf die Zündspannung der Edelgase (in German), Z. Phys. 46 (1928) 335.

[17] F.M. Penning, The starting potential of the glow discharge in neon argon mixtures between large parallel plates: II. Discussion of the ionization and excitation by electrons and metastable atoms, Physica 1 (1934) 1028.

[18] M.J. Druyvesteyn and F.M. Penning, The mechanism of electrical discharges in gases of low pressure, Rev. Mod. Phys. 12 (1940) 87 [Erratum ibid. 13 (1941) 72].

[19] G.D. Alkhazov, Ionization cascade in helium caused by nonrelativistic electrons, Zh. Tekh. Fiz. 41 (1971) 2513 [Sov. Phys. Techn. Phys. 16 (1972) 1995].

[20] I. Krajcar Bronić and B. Grosswendt, Ionization yield formation in argon-isobutane mixtures as measured by a proportional-counter method, Nucl. Instrum. Meth. B 117 (1996) 5.

[21] I. Krajcar Bronić and B. Grosswendt, Gas amplification and ionization coefficients in isobutane and argon-isobutane mixtures at low gas pressures, Nucl. Instrum. Meth. B 142 (1998) 219.

[22] K. Jelen, Avalanche multiplication of electron in the mixtures of gases and vapours (in Polish), Scientific Bulletins of the Stanislaw Staszic University of Mining and Metallurgy No 786, Craców Poland (1980).

[23] J. Zajac, Electron multiplication process in the mixtures of gases and vapours at low mixture pressure, Ph.D. thesis, AGH, University of Science and Technology, Craców Poland (1994).

[24] W. Diethorn, A methane proportional counter systems for natural radiocarbon measurements, technical report U.S. AEC NYO-6628, U.S.A. (1956).

[25] A. Zastawny, Standardization of gas amplification description in proportional counters, Nucl. Instrum. Meth. A 385 (1997) 239.

[26] Ö. Şahin, T.Z. Kowalski and R. Veenhof, High-precision gas gain and energy transfer measurements in $\mathrm{Ar}-\mathrm{CO}_{2}$ mixtures, Nucl. Instrum. Meth. A 768 (2014) 104.

[27] Ö. Şahin et al., Penning transfer in argon-based gas mixtures, 2010 JINST 5 P05002.

[28] D.R. Lide ed., Electron work function of the elements, CRC Handbook of Chemistry and Physics, internet version http://www.hbcpnetbase.com, CRC Press, Boca Raton FL U.S.A. (2005).

[29] Ö. Şahin, I. Tapan and R. Veenhof, Secondary avalanches in gas mixtures, Nucl. Instrum. Meth. A 718 (2013) 432.

[30] J. Berkowitz, Atomic and molecular photoabsorption, chapter 5, Academic Press, U.S.A. (2002), pg. 189. 